\section{Understanding Unintended Consequences and Health Information Technology:}

\section{Contribution from the IMIA Organizational and Social Issues Working Group}

\author{
C. E. Kuziemsky' R. Randell', E. M. Borycki \\ 1 Telfer School of Management, University of Ottawa, Ottawa, ON, Canada \\ 2 School of Healthcare, University of Leeds, Leeds, UK \\ 3 School of Health Information Science, University of Victoria, Victoria, BC, Canada
}

\section{Introduction}

The biomedical and health informatics communities have long been interested in the presence of unintended consequences (UICs) arising from the implementation of health information technology (HIT). Seminal work on UICs was done by Ash and colleagues, who studied them in computer physician order entry (CPOE) implementations and developed a list of UICs [1]. Various types of UICs including workflow, patient safety, communication, data entry and retrieval have been described over the past several years [1-4]. UICs have taught us that HIT evaluation needs to go beyond the task at hand and consider the wider system where HIT is used. Evaluating CPOE systems from the perspective of task automation (i.e. how well they facilitated order entry) would have produced favourable results. However, HIT automation cannot be solely evaluated by looking at the task being automated. Ripple effects from the process automation including UICs such as workflow modifications, power issues, and communication changes must be included in the evaluation.

Since this seminal research, an appreciation of the need to study UICs developed. One aspect is the recognition that different types of UICs exist. Bloomrosen et al. described different types of UICs and categorized them into a matrix [5]. Most significantly, they made a distinction between anticipated and unanticipated UICs and desirable and undesirable UICs [5].
While unintended consequences often have a negative connotation or are perceived as being bad - and indeed some studies have explicitly focused on negative UICs [4] other studies have shown that positive or beneficial UICs can occur when HIT is introduced [6-7].

While a common approach has been to identify post-HIT implementation UICs, a better strategy would be to predict them in the pre-implementation phase, and be proactive rather than reactive in their management [8]. Regardless of whether we view UICs from a positive or negative perspective, we need to better understand how UICs occur so that we can better manage them as part of HIT design and evaluation. However, realizing that HIT use is very context dependant, we must anticipate that the presence and significance of UICs will vary in different contexts [9-10]. For example, two significant contexts where HIT is used are in the organizational and social contexts. The organizational and social contexts are defined by the International Medical Informatics Association as the set of socio-technical, organizational, social, ethical, and individual behavioural issues surrounding the introduction and use of informatics applications. While there is a body of research on organizational and social issues and HIT [11-14], these issues remain underrepresented in evaluation strategies involving UICs [12, 14].

Overall, there is no framework for identifying and studying UICs with a focus on organizational and social issues. To address this shortcoming, we conducted a literature 
review and analysed the papers in order to develop a framework for considering UICs and HIT from the perspective of organizational and social issues (OSIs).

\section{Methods}

The methods we used for our literature review are largely consistent with literature review guidelines such as the ones proposed by Templier and Paré [15], who list six generic steps involved when conducting a review: 1 . formulating the research question(s) and objective(s), 2. searching the extant literature, 3. screening for inclusion, 4. assessing the quality of primary studies, 5. extracting data, and 6 . analysing data. We excluded step 4 because our intention was to map the literature rather than undertake a formal assessment of its quality.

\subsection{Study Inclusion Criteria}

Our study objective was to develop a framework for considering UICs and HIT from the perspective of OSIs. Studies were included in the review if they provided empirical (qualitative or quantitative) data on the unintended consequences of HIT.

\subsection{Search Strategy}

As the intention was to capture a broad overview of the current literature on the unintended consequences of HIT, a comprehensive literature search was not undertaken. Instead, we searched the Scopus database for January 2000 to November 2015. We conducted a literature review using the terms "health information technology" and "unintended consequences". Due to limited time and resources, the search was restricted to papers in English language and containing an abstract. A hand search of the reference lists of relevant papers was also conducted.

\subsection{Study Selection}

All retrieved records were screened based on title and abstract. Full text copies of po- tentially eligible papers were retrieved and re-screened. We retrieved 67 articles from our search. While our focus was on organizational and social aspects of UICs, adding terms such as "organizational" or "social" made the search too restricting. For example, adding the term "social" to the search criteria reduced the number of retrieved papers by approximately $75 \%$. Search results were exported into a spreadsheet and screened by two of the authors (CEK and RR). Non-empirical studies or commentaries were excluded.

\subsection{Data Extraction, Analysis, and Synthesis}

Data extracted for each paper included study design, sample type and size, setting, technology studied, key findings, and the definition used for unintended consequences. A synthesis of the extracted data was then carried out and the articles were summarized according to study design, system setting, technology used, and findings. We used the summaries to guide the analysis of the papers in order to identify themes on OSIs and UICs.

CEK and RR did the initial thematic analysis after which the themes were sent to the other co-author (EMB) for feedback and to reach consensus on the themes.

\section{Results}

From the 67 papers retrieved from Scopus, 49 papers were excluded, leaving a total of 18 papers. Papers $[16,17]$ reported on the same study as did papers [24-26] (albeit the papers all used different analytical methods or conceptual frameworks and presented findings in different ways). One paper [18] drew on the results of three different studies, while another paper [26] reported on two different studies (one of which was also reported in [24, 25]). Calculating for repeated or additional studies left 18 unique studies included in the review.

\subsection{Study Characteristics}

Table 1 provides a summary of the retrieved papers. Sixteen of the 18 papers used only qualitative approaches, with a combination of observations and interviews being the most popular study design. Two studies $[27,28]$ used mixed-methods approaches. A range of technologies were studied, including medication systems (e.g. barcode medication administration), CPOE, electronic health records (EHRs), and clinical decision support systems (CDSSs). The majority of studies $(\mathrm{N}=12)$ were undertaken in the US but there were also studies from Australia, Canada, Denmark, Israel, the Netherlands, Norway, and Tasmania.

Our analysis led to the identification of two overarching themes. The first one focuses on the definitions and terminologies used to classify and discuss UICs. The second one describes specific OSIs issues related to UICs. For the latter theme we identified four sub-themes: (1) process change and evolution, (2) individual-collaborative interchange, (3) context of use, and (4) approaches to model, study, and understand UICs. We discuss the two overarching analytical themes and accompanying sub-themes below.

\subsection{Definitions of Unintended Consequences}

Variation in how the term 'unintended consequences' was used was found among the studies. Some studies appeared to treat the term as synonymous of errors [16, 17, 24, 28], although Ash et al. [19] were clear that 'unintended consequences include more than errors'. Ash et al. [19] also provided a helpful distinction between unintended and unanticipated consequences:

'The terms "unintended consequences" and "unanticipated consequences" are not synonymous. The "unintended" implies lack of purposeful action of causation, while "unanticipated" means an inability to forecast what eventually occurred. Either kind of consequence can be adverse or beneficial. Unanticipated beneficial consequences are actually happy surprises. Unanticipated, unintended adverse consequences capture news headlines and are often what people imagine when they hear the term "unintended consequences". 
Understanding Unintended Consequences and Health Information Technology

Table 1 Papers from the review

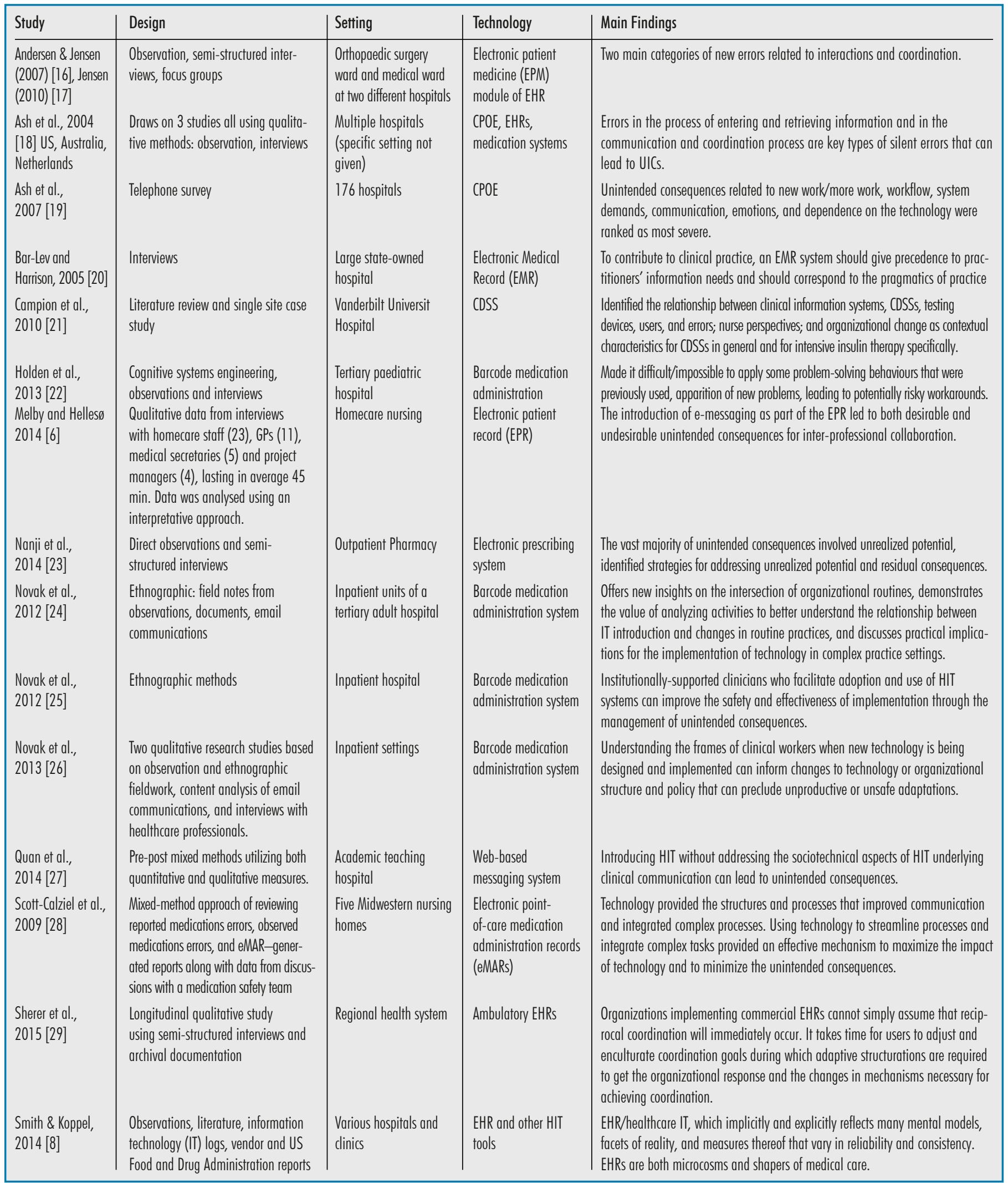




\begin{tabular}{|c|c|c|c|c|}
\hline Study & Design & Setting & Technology & Main Findings \\
\hline $\begin{array}{l}\text { Wu et al. } \\
2014 \text { [31] }\end{array}$ & $\begin{array}{l}\text { In-depth interviews and non- } \\
\text { participatory observation sessions }\end{array}$ & $\begin{array}{l}\text { General Internal } \\
\text { Medicine wards at five } \\
\text { academic teaching } \\
\text { hospitals }\end{array}$ & $\begin{array}{l}\text { Computer-mediated } \\
\text { communication } \\
\text { systems }\end{array}$ & $\begin{array}{l}\text { Text-based communication in hospital settings led to the oversimplification } \\
\text { of messages and depersonalization of communication. These unintended } \\
\text { consequences need to be recognized to avoid negative impacts to patient care } \\
\text { and work relationships. }\end{array}$ \\
\hline
\end{tabular}

\subsection{OSIs and UICs}

Our analysis of OSI themes went through several rounds. As we began our analysis, the first key finding was that organizational and social processes were a common theme, with a particular focus on how processes change and evolve over time contributing to UICs. We also identified the relationship between individual providers and collaborative teams as a source of UICs, largely due to the need to reconcile individual and collaborative processes. As the study sites and settings varied across papers, we identified organizational and social contexts as important in understanding why UICs occurred. Finally, a common theme was that prior to any attempts to mitigate UICs we need to better study and model them to gain an understanding how and why they occur. We ultimately decided upon the four OSI sub-themes listed in section 3.1 as they represent the full spectrum of studying OSIs and UICs including processes, people (i.e. individuals and teams), contexts of use, and the need for new approaches to model, study, and understand UICs and OSIs. Each of the four sub-themes is discussed below.

\subsubsection{Process Change and Evolution}

UICs can occur because HIT may alter existing processes or enable new processes. Papers described how HIT altered existing processes including communication, order entry, medical reconciliation, prescribing, diagnoses, and decision-making [17, 19, 22, $23,31]$. For example, HIT caused de-contextualization of communication processes due to the replacement of fluent synchronous conversation with asynchronous back and forth dialogue, resulting in miscommu- nication [31]. One study described how problem solving behaviours performed in a certain way in the pre-HIT system could no longer be supported after HIT implementation, resulting in clinicians engaging in risky workarounds that could compromise patient safety [22]. Studies also described that newly enabled processes such as care coordination and collaboration were initially somewhat immature and needed time to develop before the full benefit of HIT could be realized [6, 29].

HIT implementation was described as a negotiation over time between vendors and users [17, 20]. When HIT is first implemented, users may use a technology in a limited manner, with an increasing degree of process automation occurring over time [16, 29]. This longitudinal implementation should be seen as the norm and not the exception as implementing HIT and immediately expecting clinicians to use it as designed is a fallacy [26]. HIT implementation must be viewed as a mutual transformation for organizations and users, with adaptations taking place as work is transformed over time [26]. One study described how clinicians initially used an EHR system solely as a documentation tool and how clinicians enculturated and adapted coordination strategies that were enabled through the EHR over time [29].

\subsubsection{Individual-Collaborative Interchange}

A significant OSI issue is the movement from individual to collaborative processes, which we refer to as the individual-collaborative interchange. Individuals may have to work differently than they did in the past as part of the interchange [29]. While changes to work practices are often described as being detrimental to care delivery $[18,19,20,23]$, it must be emphasized that this is not always the case. Melby and Hellesø described how HIT empowered nurses, which led to beneficial changes at the group level [6]. One significant benefit from HIT is that it can facilitate communication and connectivity across providers and settings. However, HIT-mediated connectivity also brings with it new challenges and responsibilities. One such challenge is a loss of social presence in HIT-mediated communication compared to what can be achieved during face-to-face exchange [6, 31]. Even though HIT may be designed to improve communication and coordination as part of care delivery, the loss of interpersonal relations and social presence because of HIT may actually reduce interprofessional collaboration [31].

Regardless of how well HIT is designed, there will have to be trade-offs made as part of the interchange [19]. Individuals may have to give up certain features or work practices from the past system as part of collaborative design [29]. The impact of these changes can be significant including users engaging in risky workarounds and changes to how communication occurs $[6,8,22]$. It is essential these trade-offs be identified proactively so they can be managed pre-implementation.

While HIT may provide the structure and processes to improve communication, support effective decision making, and integrate complex tasks [28], it is not sufficient to just implement HIT, but rather the transition from individual to collaborative processes requires the establishment of 'rules of engagement' for how collaborative processes should be conducted [24, 31]. These 'rules' may include protocols for communication (relevance, urgency, and 
content) [31] and for coordinating collaborative tasks, such as check boxes to indicate completion of a group task [30, 31].

\subsubsection{Context of Use}

The management of UICs requires us to identify and incorporate local contexts into HIT implementation [19,24]. One study described how context is essential for ecologically valid design, which refers to an accurate understanding of how work processes are actually performed as opposed to overly simplistic assumptions of how things should be done [22]. Novak et al. point to the difference between the ostensive and performance dimensions of HIT design, with the former referring to the abstract understanding of routines while the latter refers to the reality of how HIT is actually used by different users [24]. Others described the need for localization of HIT implementation in order to fit the pragmatics of clinical practice in a particular context [20].

Some papers identified specific contexts of use. One specific context of HIT use is the network of organizational relationships [24]. The greater the network of organizational relationships that HIT works within the greater the potential for UICs [29]. One type of relationship is the number of tasks or routines that HIT intersects with. Novak et al. described how HIT-mediated changes to one routine led to UICs in its intersection with other organizational routines, even though these supplementary routines were not directly part of the HIT design [24]. A second type of relationship is at the user level. HIT that is designed for use by a collaborative team offers more potential for UICs because of the different ways users may interact with HIT [31]. In a team-based environment, it is essential that all team members abide by the norms and protocols for HIT use or else safety issues or other UICs may arise [29, 30, 31].

User involvement in HIT design was highlighted as the best way to understand contexts of use between HIT and users and work practices. Front line users are the people with the best knowledge of the context of use and they can facilitate better adoption and safer use of HIT to help with the management of UICs [24]. Further, engaging local users has been shown to actually help HIT improve clinical work [28].

\subsubsection{Need for Better Approaches to Model, Study, and Understand UICs}

A shortcoming with much of the existing work on UICs is its reactive nature as we observe the presence of UICs post-implementation and then try to deal their fallout $[8$, 18]. A first step to more proactive approaches is to formalize how we study UICs. Several studies from our review [16, 22, 24] talked about UICs in terms of "potential risks", suggesting that those UICs were not necessarily observed or reported by interviewees, but rather were the authors' reflections on what could happen. While all of our studies looked at the presence of UICs in some manner, there remain a limited number of taxonomies or models for studying them, examples of such models being $[8,19]$.

Sixteen of the 18 papers used only qualitative approaches including ethnography, observations, and interviews. Some studies used theories or models in order to explore specific details on HIT use, including Coordination Theory [29], Structuration of Technology, and Task Use Mediation models [20, 24]. Two papers used a mixed-methods approach identifying the prevalence of medication errors through incident $\operatorname{logs}$ and other supplemental data, and then using root-cause analysis to identify how the errors occurred [27, 28].

A common theme across the studies was the need to acknowledge that HIT implementation is complex [8, 22, 24, 27, 31]. Studies of HIT need to embrace that complexity by moving away from 'tidy' use cases of how HIT is used and instead focus on representing and understanding the messy and complex nature of how HIT interacts with people and processes [8]. Several studies distinguished between how HIT was designed and the system or performance dimensions of how HIT was actually used in practice [8, 20,24]. At times the gap between the design and performance dimensions was quite large and from this gap UICs emerged. Studying UICs also requires to look beyond HIT or the processes HIT is automating and to examine the web of relationships (e.g. supplementary users, processes, or technologies) that HIT may interact with during the course of care delivery. Studies identified that the way HIT enabled processes such as communication or decision-making may not be the same as in the pre-HIT era [6, 29, 31]. Understanding UICs requires us to study the change to a process that occurs because of automation, examples being alterations to social presence or media richness from the automation of communication processes [30], and weakened interpersonal relations from e-messaging [6]. Finally, while much of the existing research has focused on negative UICs, UICs can also be beneficial in that they may elicit positive changes that actually improve care delivery $[6,19]$.

\section{Discussion}

This paper identified two overarching themes: definitions of UICs, and organizational and social issues and UICS, and then discussed each theme and associated subthemes in detail. While our literature review and analysis identified a number of issues that require further research, there are two issues that our working group believes are significant research priorities. First, is the need for formal definitions of the UICs (both positive and negative) arising from the introduction of HIT. UICs are more than just errors, they also include workflow changes, emotions, and communication changes [19, 32]. Second, is the need for research that identifies and documents the outcomes of introducing HIT beyond intended benefits (i.e. as intended by technology designers such as order entry automation) by focusing on unintended consequences and unintended benefits $[6,7,19]$. In particular, there is a need for more research on positive or beneficial unintended consequences. The connectivity facilitated by HIT can enhance processes in beneficial ways beyond what was intended in system design requirements. While research on unintended benefits is limited, there are studies that have identified unintended benefits of HIT and attempted to classify them [7].

Fig. 1 summarizes the findings from our work in the form of a framework for studying UICs and HIT across the system development lifecycle from analysis to maintenance. Our framework has two as- 


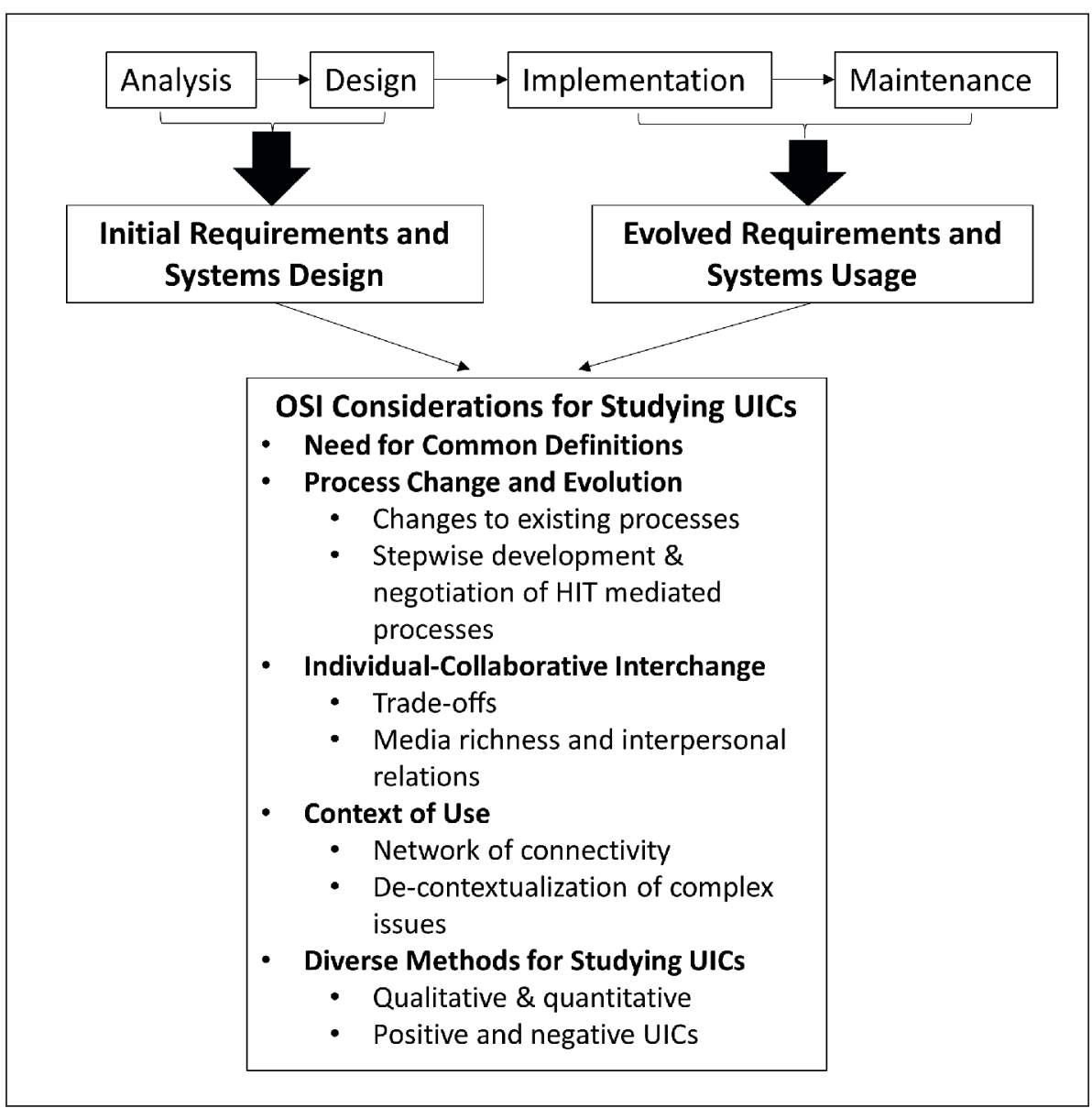

Fig. 1 Framework of OSI considerations for studying UICS and HIT

pects: the manner in which UICs need to be studied and a set of OSI considerations as basis for what should be studied. The OSI considerations were derived from the two overarching themes described earlier in this paper. The OSI considerations provide categories (e.g. Individual-Collaborative interchange, context of use) as well as specific issues (e.g. trade-offs at the individual-collaborative interchange and the network of connectivity for studying contexts of use) for each category as part of studying UICs and HIT. The top part of the framework highlights that UICs must be studied and understood both pre- and postHIT implementation. During the analysis and design phases (initial requirements and system design), UICs should be studied from the perspective of process changes, individual-collaborative interchange, and contexts of use. Once HIT is implement- ed, UICs need to be studied again in the context of how requirements might have evolved or changed after implementation (e.g. process evolution or maturity).

The OSI considerations in our framework represent research and funding priorities on behalf of our working group and also provide explicit recommendations for studying UICs and HIT. They emphasize the need for research on UICs beyond the technology itself and the need to explore how the network associated with HIT tools contributes to the development of UICs. For example, part of the management of UICs requires understanding the entire spectrum of the processes that HIT will impact. HIT may impact supplementary processes such as communication or care coordination as the process automated (e.g. order entry, medication reconciliation). Studying UICs also require us to look at the interchange between individual providers and collaborative care delivery. There is a need to understand the rules of engagement for individual work practices integration into collaborative practices, as well as the tradeoffs that individual providers have to make in the context of working collaboratively. These aspects of the individual-collaborative interchange must be studied and reconciled prior to HIT implementation.

We also need to consider social factors of HIT implementation such as social presence and interpersonal relations [6,31]. Specific contexts of use such as the network of organizational relationships must be studied as part of the understanding and management of UICs. The more tasks or users HIT interacts with, the greater the potential for UICs to emerge. A key part of understanding context of use is engaging with users in order to understand how organizational routines and relationships shape their work practices [25]. User engagement is necessary across the system development cycle not just to ensure that HIT is well designed, but also to ensure it is implemented and managed successfully in a specific context of use. Papers from our review identified how user engagement can aid the management of UICS through improved safety and effectiveness of HIT implementation and even improve clinical outcomes $[25,29]$.

Our framework also points out that UICs need to be studied using diverse methods. In particular, there is a need for more mixed-methods and quantitative research in studying UICs. Without dismissing the importance of qualitative methods, we are arguing for the use of a greater range of methods. A general weakness in the literature on UICs is the focus on qualitative methods which means that we know little about the frequency with which UICs occur. The survey by Ash et al. [19] was useful in confirming that the UICs observed in previous studies are experienced by various other hospitals. However, having identified the types of UICs that are experienced across multiple settings, it would be useful to start to quantify them in order to provide the data that is necessary for policy makers and healthcare managers to decide on priorities and resources. 
Qualitative studies are important for the initial identification of UICs from a particular technology but, using this information, we should then include the types of UICs as outcome measures in more formal, quantitative evaluations. For example, in the UK, guidance from the Medical Research Council for the design and evaluation of complex interventions [33], of which HIT would be an example, has led to acceptance that it is not appropriate to transition directly from the development and implementation of a complex intervention to a randomised controlled trial (RCT). Rather, there needs to be a series of studies, some of which will include qualitative methods, leading up to the RCT that clarifies how and in what contexts the intervention produces specific outcomes, both intended and unintended. Such knowledge can then inform what data should be gathered in the RCT. In feasibility evaluations, qualitative methods should seek to identify UICs so that they can be quantified in the definitive RCT. To fulfil the previously identified need for mixed-methods studies, methods such as simulation and agent-based modelling [34-35] provide ways to proactively identify or 'map' the web of interactions that HIT-induced change may precipitate. That 'map' would give an understanding of the breadth and depth of UICs that may exist to enable us to better position qualitative research [36].

An acknowledged shortcoming of our paper is the limited search terms we used for our literature review. Undoubtedly, we have missed papers that have described post-HIT implementation issues (including issues within the themes of this paper) that would classify as UICs but were not explicitly referred to as UICs in the publications. This further emphasizes our earlier point about the need to formalize the terminology around UICs to enable consistent tracking and reporting of them. Our framework provides such a set of terms. Another shortcoming is that we only searched for literature that used 'health information technology' as a keyword and therefore we may have missed papers on UICs that used specific HIT keywords such as CPOE or EHR.

\section{Conclusion}

HIT can introduce a wide range of UICs including issues in communication, documentation, safety, data entry, and retrieval. To date, no framework exists for identifying and studying UICs with a focus on organizational and social issues. This paper conducted a literature review on UICs and HIT and then used the findings to develop a framework of OSI considerations for studying UICs both pre- and post-implementation. Our findings and framework extend existing work on HIT and UICs by focusing on organizational and social considerations and enables HIT developers to more explicitly consider UICs during HIT design, implementation and evaluation.

\section{References}

1. Ash JS, Sittig DF, Dykstra RH, Guappone K, Carpenter JD, Seshadri V. Categorizing the unintended sociotechnical consequences of computerized provider order entry. Int J Med Inform 2007;76 Suppl 1:S21-7.

2. Harrison MI, Koppel R, Bar-Lev S. Unintended consequences of information technologies in health care: an interactive sociotechnical analysis. J Am Med Inform Assoc 2007; 14: 542-9.

3. Kuperman GJ, McGowan JJ. Potential Unintended Consequences of Health Information Exchange. J Gen Intern Med 2011;28(12):1663-6.

4. Kushniruk AW, Triola MM, Borycki EM, Stein B, Kannry JL. Technology induced error and usability: the relationship between usability problems and prescription errors when using a handheld application. Int J Med Inform 2005; 74(7):519-26.

5. Bloomrosen M, Starren J, Lorenzi NM, Ash JS, Patel VL, Shortliffe EH. Anticipating and addressing the unintended consequences of health IT and policy: a report from the AMIA 2009 Health Policy Meeting. J Am Med Inform Assoc 2011;18(1):82-90.

6. Melby L, Hellesø R. Introducing electronic messaging in Norwegian healthcare: Unintended consequences for interprofessional collaboration. Int J Med Inform 2014;83(5);343-53.

7. Kuziemsky CE, Borycki E, Nøhr C, Cummings $\mathrm{E}$. The nature of unintended benefits in health information systems. Stud Health Technol Inform 2012;180:896-900.

8. Smith SW, Koppel R. Healthcare information technology's relativity problems: a typology of how patients' physical reality, clinicians' mental models, and healthcare information technology differ. J Am Med Inform Assoc 2014; 21:117-31.

9. Scott P. Exploiting the information revolution: call for independent evaluation of the latest English national experiment. J Innov Health Inform
2015;22(1):244-9.

10. Stead WW, Lin HS (eds). Computational technology for effective health care. Washington, DC: National Academies Press; 2009.

11. Karsh BT, Weinger MB, Abbott PA, Wears RL. Health information technology: fallacies and sober realities. JAm Med Inform Assoc 2010; 17: 617-23.

12. Cresswell K, Sheikh A. Organizational issues in the implementation and adoption of health information technology innovations: an interpretative review. Int J Med Inform 2013;82(5):e73-86.

13. Lluch M. Healthcare professionals' organisational barriers to health information technologies-a literature review. Int J Med Inform 2011;80(12):849-62.

14. Kuziemsky CE. Review of social and organizational issues in health information technology. Healthc Inform Res 2015;21 (3):152-60.

15. Templier M, Paré G. A framework for guiding and evaluating literature reviews. Communications of the Association for Information Systems, 2015;37(6):112-37.

16. Andersen PER, Jensen T. Evaluating an Electronic Patient Medicine Module Implementation in two Hospital Settings: an Occasion for new Types of Errors. In: Proceedings of the European Conference on Information Management and Evaluation. Academic Conferences Limited; 2007. P. 13-20.

17. Jensen TB. Can We Rely on Electronic Medical Record Systems to Reduce Medication Errors? AMCIS 2010 Proceedings. Paper 353.

18. Ash JS, Berg M, Coiera E. Some Unintended Consequences of Information Technology in Health Care: The Nature of Patient Care Information System-related Errors. J Am Med Inform Assoc 2004;11:104-12.

19. Ash JS, Sittig DF, Poon EG Guappone K, Campbell E, Dykstra R. The extent and importance of unintended consequences related to computerized provider order entry. J Am Med Inform Assoc 2007;4(4): 415-23.

20. Bar-Lev S, Harrison MI. Localization of Health Information Technology: How Users 'Repair' Electronic Medical Systems? 2005; Academy of Management 2005 Annual Meeting: A New Vision of Management in the 21st Century, AOM 2005, $6 \mathrm{p}$.

21. Campion TR, Waitman LR, May AK, Ozdas A, Lorenzi NM, Gadd CS. Social, organizational, and contextual characteristics of clinical decision support systems for intensive insulin therapy: a literature review and case study. Int J Med Inform 2010; 9(1):31-43.

22. Holden RJ, Rivera-Rodriguez AJ, Faye H Scanlon MC, Karsh BT Automation and adaptation: nurses' problem-solving behavior following the implementation of bar-coded medication administration technology. Cogn Technol Work 2013;15(3):283-96.

23. Nanji KC, Rothschild JM, Boehne JJ, Keohane CA, Ash JS, Poon EG. Unrealized potential and residual consequences of electronic prescribing on pharmacy workflow in the outpatient pharmacy. J Am Med Inform Assoc 2014;21:481-6.

24. Novak L, Brooks, J., Gadd, C. Anders S, Lorenzi $\mathrm{N}$. Mediating the intersections of organizational routines during the introduction of a health IT system. Europ J Inf Syst 2012; 21(5).

25. Novak LL, Anders S, Gadd CS, Lorenzi NM. 
Mediation of adoption and use: a key strategy for mitigating unintended consequences of health IT implementation. J Am Med Inform Assoc 2012; 19 (6): 1043-4049.

26. Novak LL, Holden RJ, Anders SH, Hong JY, Karsh B.-T. Using a sociotechnical framework to understand adaptations in health IT implementation. Int J Med Inform 2013;82 (12): e331-e344.

27. Quan SD, Wu RC, Rossos PG, Arany T, Groe S, Morra D, et al. It's not about pager replacement: An in-depth look at the interprofessional nature of communication in healthcare. J Hosp Med 2013; Mar;8(3):137-43.

28. Scott-Cawiezell J, Madsen RW, Pepper GA, Vogelsmeier A, Petroski G, Zellmer D. Medication safety teams' guided implementation of electronic medication administration records in five nursing homes Jt Comm J Qual Patient Saf 2009;35:29-35.

29. Sherer SA, Meyerhoefer CD, Sheinberg M, Levick D. Integrating commercial ambulatory electronic health records with hospital systems: An evolutionary process. Int J Med Inform 2015;
Sep;84(9):683-93.

30. Wong MC, Cummings E, Turner, P. User-centered design in clinical handover: Exploring post-implementation outcomes for clinicians. Stud Health Technol Inform 2013; 192 (1-2):253-7.

31. Wu R, Appel L, Morra D, Lo V, Kitto S, Quan S. Short message service or disService: Issues with text messaging in a complex medical environment. Int J Med Inform 2014; 83:278-84.

32. Borycki EM, Kushniruk AW, Bellwood P, Brender J. Technology-induced Errors. Methods Inf Med 2012;51(2):95-103.

33. Craig P, Dieppe P, Macintyre S, Michie S, Nazareth I, Petticrew M: Developing and evaluating complex interventions: the new Medical Research Council guidance. BMJ 2008;337:a1655.

34. Nilsson F, Darley V. On complex adaptive systems and agent-based modelling for improving decision-making in manufacturing and logistics settings: Experiences from a packaging company. International Journal of Operations and Production Management. 2006;26 (12):1351-73.
35. Zhou Y, Ancker JS, Upadhye M, McGeorge NM,Guarrera TK, Hegde S, et al. The impact of interoperability of electronic health records on ambulatory physician practices: a discrete-event simulation study. Inform Prim Care 2013;21(1):21-9.

36. Borycki EM, Kushniruk AW, Carvalho CJ, Kuo $\mathrm{MH}$. A systematic review of qualitative and quantitative methods used to identify and study technology-induced errors in computerized physician order entry (CPOE). Asia Pacific Association for Medical Informatics (APAMI) 2009; November 22-26:32-7.

Correspondence to:

Craig Kuziemsky

Telfer School of Management

University of Ottawa

Ottawa, ON, Canada

Tel: +1 6135625800 ext 4792

E-mail: Kuziemsky@telfer.uottawa.ca 